\title{
Interactive comment on "A novel causal structure-based framework for comparing basin-wide water-energy-food-ecology nexuses applied to the data-limited Amu Darya and Syr Darya river basins" by Haiyang Shi et al.
}

\section{Anonymous Referee \#2}

Received and published: 9 November 2020

\section{Summary}

The study compares watersheds by assessing causality links of different variables within the Water-energy-food-ecosystem nexus by using Bayesian Network. The framework is applied to two river basins linked to the Aral Sea, to identify factors and strategies that might explain or solve trade-offs among the different sectors and actors. The approach is of interest for the reader of HESS and Innovative. The strength of the framework is to find solutions without assuming the relationship between systems (compared to process-based models); by its design it is stakeholder and data driven.

Printer-friendly version

Discussion paper 
The work is well illustrated with clear figures. Three main points could considerably increase the impact of the work: A - the methodology should be better described and eventually made available in order to be reproducible, B - it could be clearly stated what insights are gained from the framework and what are the limitations, $\mathrm{C}$ - the data used for the study should be better described and made available to the reader (as seven co-authors solely contributed on the data, it is expected to be a major contribution of this work). The following points describe the concern in detail and address a list of other minor and or important points:

A Methodology: It is not clear how this Bayesian Network is constructed, what is optimized or simulated. The authors only clearly describe 2 indicators of causality (VB and $\mathrm{MI}$ ), and 3 performance indicators (/objectives ?): reliability, total benefits and cooperation, the rest of the framework is vague.

A1 The two following describe steps general concept of the framework, however, it is later not clearly explained how those are concretely implemented - with references to the methodology/literature. I 115. "We construct a same WEFE nexus causality structure for the river basins selected in the previous step, which can be represented by a directed graph model such as the Bayesian network" I 121. "we combine the causal structure representing expert knowledge from multiple fields with actual statistics and observation data to update the initial understanding of causality. In this way, the original qualitative causal structure is quantified by actual data, and the originally scattered actual data is closely connected by the causal structure." A2 I 196. "The responsibility for exploring the differences between the two river basins mainly relies on the continuous updates of new input cases" What are new input cases? additional data? A3 I 208. "The index variance of belief (VB) and the index mutual information (MI) based on the change of information entropy (Barton et al., 2008; Marcot, 2012) - are applied to evaluate the change in strength and uncertainty of the causal relation between the nodes." explain better what those 2 indicators mean, how they can be interpreted ? A4 I 225. " We utilized the posterior probability prediction function of BN so as to support the 
decision optimization." how are "posterior probability prediction function" formulated ? - reference ? how is the "optimization" formulated - what are the variables - objective

- reference? A5 In general, it is common in the HESS journal that authors if possible, provide the code, software files, so that others can use the framework. It would clearly increase the impact of the work to share the programming tools that were used (in a re-usable way).

B How does the framework guide decision making ?

B1 I 378. "In addition to the widely recognized differences in glacier melting in high mountainous areas, this study shows that the ratio of the upstream reservoir interception water to the total runoff is largely different in these two river basins" Do we need to apply the methodology to reach this conclusion? is the data on runoff and reservoir capacity not already showing this ? B2 The rest of the promising solutions presented in sections 5.2 and 5.3 are not based on the framework, at least not to my understanding. If yes, then explained how the framework leaded to find such solutions. If not, why did the framework not help to identify those solutions? How could we improve it to do so? B3 I 444. "It might characterize the hidden uncertainty in the decision support." what hidden uncertainty has this study revealed ? B4 The limitations of the framework could be more clearly stated. In which cases will the framework fail to identify sources of problems. B5 Maybe it would be easier to understand if the authors clearly differentiated between two steps: What insights does the framework give when applying the $\mathrm{BN}$ to a single river basin (/case) regarding causality and management options? What insights come from the comparison of the causality links between two cases/river basins? How can one basin learn from another by looking at causality links? (what insights cannot be found by looking only at one basin) and also: How does that compare to insights found by process based model as mentioned by the authors? what are advantages and disadvantages?

Printer-friendly version

Discussion paper

C Data 
C1 I 465. The authors state: "The data sources we used in this study have been listed in the main text. Data can also be obtained by requesting the corresponding author." The authors are referred to the guidelines of the journal regarding data: https://www.hydrology-and-earth-system-sciences.net/policies/data_policy.html In table 2, the reference to the data is not precise enough, general websites are indicated. Some links do not work, e.g. https://www.cawater-info.net needs http and not https. The data should be published in a data repository with some decent meta-data level. Seven of the co-authors contribution is solely on data, hence I expect the dataset to be a major contribution of this work, thus special attention should be payed to it.

\section{D other comments}

D1 I 22. Water-energy-food nexus or nexuses ? Inconsistent through manuscript, most literature choose nexus D2 I 22-23. the river basins did not cause the Aral sea disaster, but poor water management did. D3 I 78. many empirical parameters : give examples D4 I 89. the word "superiority" might be overclaimed. It would be also interesting to describe what type of outcomes are available from the different studies. D5 I 131. not clear what that "etc." refers to: remove. D6 I 227. "we selected the scenarios" which scenarios are we talking about? D7 I 253. why use "international trade" market prices, what for ecosystems? D8 I 277-279. and Figure 8. "During the period 1980 - 1991, the contribution of most variables has declined, which may be related to the normalization of the maximized agricultural production, leaving only the natural runoff as the main variation contribution. "Should the sum of the contribution of all variables not match a $100 \%$ ? it is stayed that the runoff becomes the main variation contribution, however also the runoff variable has a decreasing value in the VB ratio. So what is the variable that increases if all the variables showed decrease? D9 I 311. reducing flow to depressions is presented as the best solution, but in the previous section it is described as generating trade-off with other sectors and ecosystems - explain. D10 I 315. the term "positive" might be misleading, it seems drought have a positive effect on salinization, desertification in the sense "good", "desirable" ...

Printer-friendly version

Discussion paper 
Interactive comment on Hydrol. Earth Syst. Sci. Discuss., https://doi.org/10.5194/hess-2020482, 2020.

Interactive

comment 